\title{
Umbilic and Tangential Singularities on Configurations of Principal Curvature Lines
}

\author{
RONALDO GARCIA ${ }^{1}$ and JORGE SOTOMAYOR ${ }^{2}$ \\ ${ }^{1}$ Instituto de Matemática e Estatística - Universidade Federal de Goiás, Cx. Postal 131 \\ 74001-970 Goiânia, GO, Brazil \\ ${ }^{2}$ Instituto de Matemática e Estatística, Universidade de São Paulo, Cidade Universitária \\ 05508-900 São Paulo, SP, Brazil \\ Manuscript received on June 20, 2001; accepted for publication on October 18, 2001; \\ contributed by JoRge Sotomayor
}

\begin{abstract}
In this paper is studied the relationship between quadratic tangencies of principal lines with the boundary of a surface and the Darbouxian umbilics of a smooth boundaryless surface which approximates it through the process of thickening and smoothing defined here.
\end{abstract}

Key words: tangential singularity, umbilic point, principal configuration.

\section{INTRODUCTION}

Consider a smooth i.e. $C^{\infty}$, compact, connected, oriented and regular surface $\mathbf{S}$ with boundary $\mathbf{B}$ embedded into $\mathbb{R}^{3}$. Without loss of generality, we can assume that $\mathbf{S}$ is contained in a boundaryless surface $\hat{\mathbf{S}}$ defined implicitly by a smooth function $f: \mathbb{R}^{3} \rightarrow \mathbb{R}$ such that

$$
\hat{\mathbf{S}}=\left\{p \in \mathbb{R}^{3}: f=0\right\}
$$

and that $\mathbf{S}$ and $\mathbf{B}$ are given in terms of a smooth function $b: \mathbb{R}^{3} \rightarrow \mathbb{R}$, by

$$
\mathbf{S}=\{p \in \hat{\mathbf{S}}: b \geq 0\}
$$

and

$$
\mathbf{B}=\{p \in \hat{\mathbf{S}}: b=0\},
$$

with $\nabla f \neq 0$ on $\hat{\mathbf{S}}$ and $\hat{T}=\nabla f \wedge \nabla b \neq 0$ on $\mathbf{B}$.

Correspondence to: Ronaldo Garcia

E-mail: ragarcia@mat.ufg.br 
The function $f$ is defined so that the positive orientation i.e. the positive unit normal map of the surface $\mathbf{S}$ is given by $N=\frac{\nabla f}{|\nabla f|}$. The positive orientation on $\mathbf{B}$ is defined by the unit tangent vector field $T=\frac{\hat{T}}{|\hat{T}|}$.

The principal configuration on the pair $(\mathbf{S} ; \mathbf{B})$ consisting of the oriented surface $\mathbf{S}$ and the oriented boundary $\mathbf{B}$ is defined by the quadruple

$$
\mathcal{P}_{(\mathbf{S}, \mathbf{B})}=\left(\mathcal{U}_{\mathbf{s}}, \mathcal{C}_{\mathbf{S}}, \mathcal{F}_{1 \mathbf{s}}, \mathcal{F}_{2 \mathbf{S}}\right),
$$

where $\mathcal{U}_{\mathbf{S}}$ is the set of umbilic points of $\mathbf{S}$, i.e. points at which the principal curvatures coincide i.e. $d N \equiv-k I$ for some $k, \mathcal{C}_{\mathbf{S}}$ is the set of tangential singularities on $\mathbf{B}$ at which $T$ is a principal direction, that is $d N(T)$ is collinear with $T$, and $\mathcal{F}_{1 \mathbf{S}}$ and $\mathcal{F}_{2 \mathbf{S}}$ are the minimal and maximal principal foliations on $\mathbf{S} \backslash \mathcal{U}_{\mathbf{S}} \cup \mathcal{C}_{\mathbf{S}}$. The leaves of these foliations are the arcs of minimal and maximal principal lines on $\mathbf{S} \backslash \mathcal{U}_{\mathbf{S}} \cup \mathcal{C}_{\mathbf{S}}$. The points on $\mathcal{C}_{\mathbf{S}}$ further split into $\mathcal{C}_{1 \mathbf{S}}$ and $\mathcal{C}_{2 \mathbf{S}}$ according to which foliation is tangent to $\mathbf{B}$. The arcs of $\mathcal{F}_{i \mathbf{S}}$ that touch $\mathbf{B} \backslash \mathcal{C}_{\mathbf{S}}$ are closed at their extremes located on $\mathbf{B}$.

Examples can be given easily by choosing $f$ and $b$. For instance, if $f=1-x^{2}-y^{2}, \hat{\mathbf{S}}$ is a cylinder around the $z$-axis; since the orientation is towards the outside, its minimal principal lines are the circular parallels and its maximal principal ones are the meridians, which are lines parallel to the $z$-axis. Taking $b=r^{2}-y^{2}-z^{2}$, for $r<1$, get two cylindrical disks. Each one has four tangential singularities, two minimal and two maximal, all external. For $r>1$, get a cylindrical surface whose boundary consists of two sinusoidal closed curves, each of which carries two internal and two external minimal tangencies. Notice that there is no maximal tangential singularities in this example.

The examples above do not have umbilic points. By taking $\hat{\mathbf{S}}$ to be an ellipsoid with three different axes, examples with umbilic points can be obtained.

The principal configuration $\mathcal{P}_{(\mathbf{S}, \mathbf{B})}$ carries the extremal - maximal and minimal - bending structure of a surface. In Surface Theory it is the natural analogous to the phase portrait which, in Differential Equations and Dynamical Systems, carries the orbit structure of a vector field or flow (Melo and Palis 1982). For the case of boundaryless surfaces the principal configuration has been first studied by (Gutierrez and Sotomayor 1982, 1991) from the point of view of Structural Stability under small deformations of $\mathbf{S}$. It, however, has deep roots that goes back to the classical works of Monge, Dupin and Darboux, (Darboux 1896) and (Gutierrez and Sotomayor 1991). See also (Gutierrez and Sotomayor 1998), for a survey on Charathéodory conjecture on umbilic points on convex surfaces and recent developments on structural stability. For an extension of principal configurations to surfaces in $\mathbb{R}^{4}$, see the paper (Garcia and Sotomayor 2000).

The reader is referred to (Spivak 1980) for the basic general properties of lines of curvature and umbilic points.

A number of natural mathematical questions could be raised now about $\mathcal{P}_{(\mathbf{S}, \mathbf{B})}$ and its dependence on deformations of the pair $(\mathbf{S}, \mathbf{B})$, i.e. on deformations of the functions $f$ and $b$. This would lead to an extension to surfaces with boundary of the structural stability results established forboundaryless ones in (Gutierrez and Sotomayor 1982, 1991). Another related sort of deformations 
of a surface with boundary will be considered in this paper.

Here will be given a first step towards the extension to surfaces with boundary $(\mathbf{S}, \mathbf{B})$ of the analysis and stability properties of principal configurations known for boundaryless surfaces. This is done by comparison with the principal configuration of a suitable deformation $\mathbf{S}_{\epsilon, \delta}$, obtained through the operations of $\epsilon$-thickening and $\delta$-smoothing applied to the surface with boundary.

The $\epsilon$-thickening of $\mathbf{S}$ is the tubular neighborhood $\mathbf{T} \mathbf{S}_{\epsilon}$ of $\mathbf{S}$ :

$$
\mathbf{T S}_{\epsilon}=\left\{q \in \mathbb{R}^{3}:|q-p| \leq \epsilon, \quad p \in \mathbf{S}\right\}
$$

For $\epsilon$ small, $\mathbf{S}_{\epsilon}=\partial \mathbf{T} \mathbf{S}_{\epsilon}$ is the envelope of the family of spheres of radius $\epsilon$ and center ranging on $\mathbf{S}$; it is a $C^{1}$ compact boundaryless surface oriented towards the exterior of $\mathbf{S}_{\epsilon}$.

This surface is smooth on the complement of the curve $\mathbf{B}_{\epsilon, \pm}$ which is the inverse image of $\mathbf{B}$ by the tubular projection $\pi: \hat{\mathbf{S}}_{\epsilon} \rightarrow \hat{\mathbf{S}}$. In fact $\mathbf{B}_{\epsilon, \pm}$ is the common border of the two connected smooth surfaces with boundary $\mathbf{S}_{\epsilon, \pm}=\mathbf{S} \pm \epsilon N$ (with a connected component for each sign) and half of the tube centered along $\mathbf{B}$ and radius $\epsilon$, defined parametrically by

$$
\mathbf{T}_{\epsilon}=\mathbf{B}+\epsilon \cos \theta N+\epsilon \sin \theta N \wedge T, \quad 0 \leq \theta \leq \pi .
$$

See Figure 1 for an illustration of the surface $\mathbf{S}_{\epsilon}$.

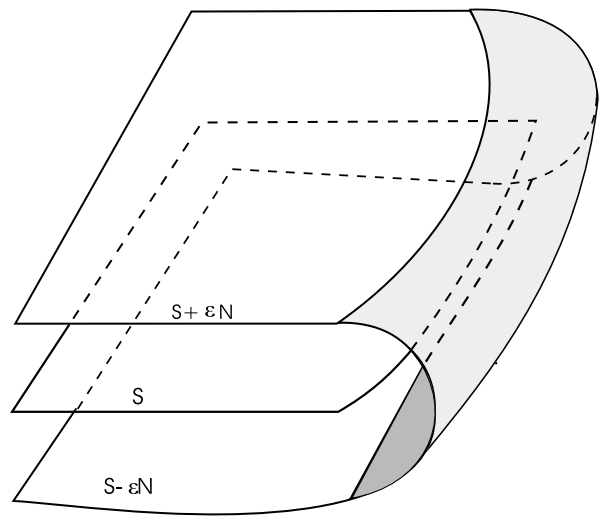

Fig. 1 - Thickening of the surface $\mathbf{S}$.

The $\delta$-smoothing of $\mathbf{S}_{\epsilon}$ is an embedding of $\mathbf{S}_{\epsilon}$ into $\mathbb{R}^{3}$ whose image is a smooth surface $\mathbf{S}_{\epsilon, \delta}$ which coincides with the identity outside a $\delta$-tubular neighborhood of $\mathbf{B}_{\epsilon, \pm}$.

This surface $\mathbf{S}_{\epsilon, \delta}$ is (not uniquely) defined by averaging the functions

$$
P_{\epsilon}=\left(x-\pi_{\hat{\mathbf{S}}}(x)\right)^{2}-\epsilon^{2}, \quad \text { for } x \text { on } \quad b \circ \pi_{\hat{\mathbf{s}}} \geq 0,
$$

and

$$
p_{\epsilon}=\left(x-\pi_{\mathbf{B}}(x)\right)^{2}-\epsilon^{2}, \quad \text { for } x \text { on } \quad b \circ \pi_{\hat{\mathbf{s}}} \leq 0,
$$

by means of a smooth transition function $h_{\delta, \epsilon}=h(\delta / \epsilon)$. 
More precisely, take

$$
P_{\epsilon, \delta}=h_{\delta, \epsilon} P_{\epsilon}+\left(1-h_{\delta, \epsilon}\right) p_{\epsilon}
$$

and $\mathbf{S}_{\epsilon, \delta}=P_{\epsilon, \delta}^{-1}(0)$ where $h$ is a smooth decreasing function in the interval $[0, \delta]$ such that $h^{(n)}(0)=h^{(n)}(\delta)=0$ for all $n \in \mathbb{N}, h(t)=1$ for $t \leq 0$ and $h(t)=0$ for $t \geq \delta$.

The principal configuration of the surface $\mathbf{S}_{\epsilon}$ outside $\mathbf{B}_{\epsilon, \pm}$ is known. On one portion it is provided by that of the Tube (Gutierrez and Sotomayor 1991); on the other, i.e. on $\mathbf{S}-\epsilon \mathbf{N}$ and $\mathbf{S}+\epsilon \mathbf{N}$, it is obtained from that of $\mathbf{S}$ by translation along $\epsilon \mathbf{N}$ and $-\epsilon \mathbf{N}$. Notice that since $\mathbf{S}_{\epsilon}$ is oriented by its outward normal, the minimal and maximal principal foliation are exchanged in the transition from $\mathbf{S}-\epsilon \mathbf{N}$ to $\mathbf{S}+\epsilon \mathbf{N}$. For the sake of completeness, the principal configurations on the Tube and translated surfaces $\mathbf{S} \pm \epsilon N$ are reviewed and established in Section 2 .

In this paper it will be proved that, with specific restrictions on the type of transition function $h$ (see section 3), from points of $\mathcal{C}_{\mathbf{S}}$ of quadratic tangencies of principal lines with $\mathbf{B}$, bifurcate only Darbouxian umbilic points of types $D_{1}$ and $D_{3}$ on $\mathbf{S}_{\epsilon, \delta}$, for $\epsilon$ and $\delta$ small. See (Gutierrez and Sotomayor 1982, 1991) and Section 4 for the basic properties of Darbouxian Umbilics.

The main result is the following.

THEOREM 1. Consider a point $p_{0}$ of $\mathfrak{C}_{\mathbf{S}}$ such that the minimal (or maximal) principal foliation has quadratic contact (internal or external cases) with the boundary at this point. Then, for appropriate transition function $h$, there exists a regular curve of umbilics, tangent to $N\left(p_{0}\right)$ and intersecting transversally the surface $\mathbf{S}_{\epsilon, \delta}$ at a point $p_{\epsilon}$, for $\epsilon>0$ (or $\left.\epsilon<0\right)$ and $\delta$ small. This point is a Darbouxian umbilic point for the surface $\mathbf{S}_{\epsilon, \delta}$ and it is of type $D_{1}$ (external tangency case) or $D_{3}$ (internal tangency case). See Figure 2.
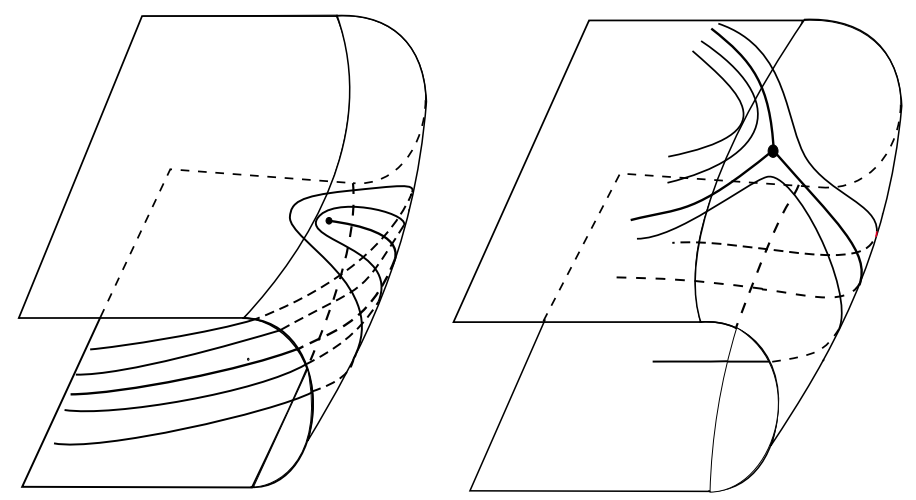

Fig. 2 - Minimal principal foliation of $\mathbf{S}_{\epsilon, \delta}$ : external and internal tangency.

This result expresses the bifurcation phenomenon of transition of tangencies into umbilics on principal configurations, under thickening and smoothing. For small $\epsilon$ and $\delta$, tangencies are transferred into umbilics, from $\partial \mathbf{S}$ to $\mathbf{S}_{\epsilon, \delta}$.

Together with umbilics, periodic lines of principal curvature, which are the compact leaves of the principal foliations, are of great relevance for the understanding of the global structure of 
principal configurations. In the forthcoming paper (Garcia and Sotomayor 2001), is pursued the study of the transition from $\mathcal{P}_{(\mathbf{S}, \mathbf{B})}$ into periodic principal lines on $\mathbf{S}_{\epsilon, \delta}$.

From the geometric point of view this work represents a contribution to the analysis of the smooth transition between the two possible principal configurations (one for each orientation) on surfaces with boundary, when passing from one orientation to the other. It is also related to the study of the transition on the phase portrait of a discontinuous differential equation (that of the maximal and minimal curvature lines, one on each side of the surface with boundary), when crossing a line of discontinuity (represented here by the boundary curve B). See the paper by (Sotomayor and Teixeira 1998) dealing with the regularization, i.e. the smoothing, of general discontinuous differential equations.

The fact that the differential equations of principal lines have a geometric realization, one on each side of the surface that carries them, makes it natural to express geometrically the smooth transition between them by means of the surface obtained with the operations of thickening and smoothing defined here.

\section{PRINCIPAL CONFIGURATION OF $S_{\epsilon}$}

This section provides a self sufficient presentation of the elementary properties of principal configurations on surfaces obtained through the thickening procedure from one with boundary.

Let $(\mathbf{S}, \mathbf{B})$ be a given surface with boundary, positively oriented by the normal unitary field $N$, as above. Consider the family of parallel surfaces $\mathbf{S}_{\epsilon}$ given by $\mathbf{S}_{\epsilon}: \mathbf{S}+\epsilon N$.

Near a connected component of the border $\mathbf{B}$ we consider a tube of radius $\epsilon$ with center ranging along $\mathbf{S}$, as in Figure 1. This procedure defines a boundaryless surface $\mathbf{S}_{\epsilon}$ of class only $C^{1}$.

Proposition 1. Let $c:[0, l] \rightarrow \mathbb{R}^{3}$ be a regular arclength parametrization of a connected component of $\mathbf{B}$, such that $\{T, N \wedge T, N\}$ is a positive frame of $\mathbb{R}^{3}$. Then the expression

$$
\alpha(u, v)=c(u)+v(N \wedge T)(u)+\left[\frac{1}{2} k_{n}^{\perp}(u) v^{2}+o\left(v^{2}\right)\right] N(u), \quad-\delta<v<\delta
$$

where $k_{n}^{\perp}$ is the normal curvature of $\mathbf{S}$ in the direction of $N \wedge T$, defines a local $C^{\infty}$ chart on the surface $\hat{\mathbf{S}}$ defined in a small tubular neighborhood of $c$.

Proof. The map $\alpha(u, v, w)=c(u)+v(N \wedge T)(u)+w N(u)$ is a local diffeomorphism in a neighborhood of the $u$ axis. For each $u$, the curve $v \rightarrow v(N \wedge T)(u)+w(u, v) N(u)$ is the intersection of the surface $\mathbf{S}$ with the plane spanned by $\{(N \wedge T)(u), N(u)\}$. Using Hadamard's lemma it follows that

$$
w(u, v)=\left[\frac{1}{2} k_{n}^{\perp}(u) v^{2}+v^{2} A(u, v)\right] N(u)
$$

where $A(u, 0)=0$ and $k_{n}^{\perp}$ is the (plane) curvature of the curve in the plane spanned by $\{N \wedge T, N\}$, that cuts the surface $\hat{\mathbf{S}}$. This ends the proof. 
REMARK 1. A similar chart has proved to be useful in (Gutierrez and Sotomayor 1982), for the study of periodic principal lines, and in (Garcia and Sotomayor 1997), for the analysis of asymptotic lines near parabolic curves.

According to (Spivak 1980), the Darboux frame $\{T, N \wedge T, N\}$ along $\mathbf{B}$ satisfies the following system of differential equations:

$$
\begin{aligned}
T^{\prime} & =k_{g} N \wedge T+k_{n} N \\
(N \wedge T)^{\prime} & =-k_{g} T+\tau_{g} N \\
N^{\prime} & =-k_{n} T-\tau_{g}(N \wedge T)
\end{aligned}
$$

where $k_{n}$ is the normal curvature, $k_{g}$ is the geodesic curvature and $\tau_{g}$ is the geodesic torsion of the boundary curve $\mathbf{B}$.

Proposition 2. Consider a surface $\mathbf{S}$ and a connected component of the boundary $\mathbf{B}$ parametrized by $c$. Then the principal lines of $\mathbf{S}$ are transversal to $c$ at a point $c\left(u_{0}\right)$ if and only if $\tau_{g}\left(u_{0}\right) \neq 0$. Assuming that $\left(k_{n}^{\perp}-k_{n}\right)\left(u_{0}\right)=\left(k_{2}-k_{1}\right)\left(u_{0}\right)>0$, a minimal principal curvature line has quadratic contact with $c$ at a point $c\left(u_{0}\right)$ if and only if $\tau_{g}\left(u_{0}\right)=0$ and $\tau_{g}^{\prime}\left(u_{0}\right) \neq 0$.

The contact is internal (respectively external) if $\tau_{g}^{\prime}\left(u_{0}\right)>0$ (respectively $\left.\tau_{g}^{\prime}\left(u_{0}\right)<0\right)$.

Proof. Using the parametrization defined by equation 1 and the Darboux frame given by equation 2, it follows that:

$$
\begin{aligned}
& E(u, 0)=1, \quad F(u, 0)=0, \quad G(u, 0)=1 \\
& e(u, 0)=k_{n}(u), \quad f(u, 0)=\tau_{g}(u), \quad g(u, 0)=k_{n}^{\perp}(u)
\end{aligned}
$$

Therefore the tangent vector $T(u)$ is a principal direction if and only if $\tau_{g}(u)=0$.

From the differential equation of curvature lines

$$
(F g-G f) d v^{2}+(E g-G e) d u d v+(E f-F e) d u^{2}=0,
$$

see (Gutierrez and Sotomayor 1991) and (Spivak 1980), it follows that near a point of tangency the minimal principal curvature lines are the solutions of the following differential equation:

$$
\begin{aligned}
u^{\prime} & =-\left(k_{n}^{\perp}-k_{n}\right)\left(u_{0}\right)+\cdots \\
v^{\prime} & =\tau_{g}^{\prime}\left(u_{0}\right)\left(u-u_{0}\right)+\cdots
\end{aligned}
$$

Therefore, $v(u)=-\frac{1}{2} \tau_{g}^{\prime}(0)\left(k_{n}^{\perp}-k_{n}\right)^{-1}(0)\left(u-u_{0}\right)^{2}+\cdots$.

REMARK 2. For the maximal principal lines the contact is internal (respectively external) if $\left(k_{n}^{\perp}-k_{n}\right) \tau_{g}^{\prime}\left(u_{0}\right)<0$ ( respectively $\left.\left(k_{n}^{\perp}-k_{n}\right) \tau_{g}^{\prime}\left(u_{0}\right)>0\right)$.

Proposition 3. Consider a surface $\mathbf{S}$ parametrized near a connected component of the boundary by $\alpha$ as in equation (1). Let $\mathbf{S}_{\epsilon,+}$ be the parallel surface defined by

$$
\alpha_{\epsilon}=\alpha+\epsilon N \text {, }
$$


Then the principal configurations of $\mathbf{S}$ and $\mathbf{S}_{\epsilon,+}$ are the same, i.e., by a parallel displacement the principal curvature lines, the umbilic points and the tangencies are preserved.

Proof. Direct calculation in any chart $(u, v)$ shows that the coefficients of the first and second fundamental forms of $\mathbf{S}$ and $\mathbf{S}_{\epsilon,+}$ are expressed as follows:

$$
\begin{aligned}
E_{\epsilon} & =(1-\epsilon \mathcal{K}) E+\left(\epsilon^{2} \mathcal{H}-2 \epsilon\right) e \\
F_{\epsilon} & =(1-\epsilon \mathcal{K}) F+\left(\epsilon^{2} \mathcal{H}-2 \epsilon\right) f \\
G_{\epsilon} & =(1-\epsilon \mathcal{K}) G+\left(\epsilon^{2} \mathcal{H}-2 \epsilon\right) g \\
e_{\epsilon} & =(1-\epsilon \mathcal{H}) e+\epsilon \mathcal{K} E \\
f_{\epsilon} & =(1-\epsilon \mathcal{H}) f+\epsilon \mathcal{K} F \\
g_{\epsilon} & =(1-\epsilon \mathcal{H}) g+\epsilon \mathcal{K} G
\end{aligned}
$$

Here $\mathcal{K}$ and $\mathcal{H}$ are respectively the Gaussian and Mean Curvature of the surface $\mathbf{S}$. Therefore,

$$
\begin{aligned}
F_{\epsilon} g_{\epsilon}-G_{\epsilon} f_{\epsilon} & =\left(1+\epsilon^{2} \mathcal{K}-\epsilon \mathcal{H}\right)(F g-G f) \\
E_{\epsilon} g_{\epsilon}-G_{\epsilon} e_{\epsilon} & =\left(1+\epsilon^{2} \mathcal{K}-\epsilon \mathcal{H}\right)(E g-G e) \\
E_{\epsilon} f_{\epsilon}-F_{\epsilon} e_{\epsilon} & =\left(1+\epsilon^{2} \mathcal{K}-\epsilon \mathcal{H}\right)(E f-F e)
\end{aligned}
$$

Therefore, differential equations of the principal curvature lines of $\mathbf{S}$ and $\mathbf{S}_{\epsilon,+}$ are the same. This ends the proof.

REMARK 3. As above, analogous identification of principal configurations, but taking into account the exchange of maximal into minimal and vise-versa, holds for the surface $\mathbf{S}$ and that defined by negative translation: $\alpha_{\epsilon}=\alpha-\epsilon N$. This is due to the orientation convention assumed.

Proposition 4. Let $c:[0, l] \rightarrow \mathbb{R}^{3}$ be a parametrization by arclength $u$ of a connected component of $\mathbf{B}$ such that $\{T, N \wedge T, N\}$ is a positive basis of $\mathbb{R}^{3}$. Then the expression below

$$
\beta(u, \theta)=c(u)+\epsilon \cos \theta N(u)+\epsilon \sin \theta(N \wedge T)(u)
$$

is a regular parametrization of the tube $\mathbf{T}_{\epsilon}$ of radius $\epsilon$ centered at the curve $c$.

The differential equation of the principal curvature lines is given by

$$
d u\left(d \theta-\tau_{g}(u) d u\right)=0 .
$$

At the points where $\tau_{g}\left(u_{0}\right)=0$ and $\tau_{g}^{\prime}\left(u_{0}\right) \neq 0$ the contact between the maximal principal lines and the curves defined by $\theta=0$ and $\theta=\pi$ is of quadratic type.

Proof. The tube centered at $c$ and of constant radius $\epsilon$ is clearly a regular surface for $\epsilon>0$ small.

Using the Darboux frame given by equation (2) and the parametrization (4), it follows that

$$
\begin{aligned}
\beta_{u} & =\left(1-\epsilon k_{n} \cos \theta-\epsilon \sin \theta\right) T-\epsilon \tau_{g} \cos \theta N \wedge T-\epsilon \tau_{g} \sin \theta N \\
\beta_{\theta} & =-\epsilon \sin \theta N+\epsilon \cos \theta N \wedge T \\
\beta_{u} \wedge \beta_{\theta} & =\epsilon\left(1-\epsilon k_{n} \cos \theta-\epsilon \sin \theta\right)[\sin \theta N \wedge T+\cos \theta N]
\end{aligned}
$$


So the coefficients of the first fundamental form are given by:

$$
\begin{aligned}
& E=\left(1-\epsilon k_{n} \cos \theta-\epsilon \sin \theta\right)^{2}+\left(\epsilon \tau_{g}\right)^{2} \\
& F=-\epsilon^{2} \tau_{g} \\
& G=\epsilon^{2}
\end{aligned}
$$

As the tube is the envelope of an one parameter family of spheres of radius $\epsilon$ centered along $c$ the circles parametrized by $u=c t e$ are the minimal principal lines, see (Gutierrez and Sotomayor 1991) and (Spivak 1980). Here the positive orientation of the tube is defined by the exterior normal, having the circles $u=c t e$ principal curvature $k_{1}=-\frac{1}{\epsilon}$. Therefore, the orthogonal family of curves, the other family of principal lines, is defined by the integral curves of the vector field $X=G \frac{\partial}{\partial u}-F \frac{\partial}{\partial \theta}$ or equivalently by the differential equation $\frac{d \theta}{d u}=-\frac{F}{G}=\tau_{g}(u)$. A solution of the equation above has the following Taylor expansion

$$
\theta(u)=\theta_{0}+\frac{1}{2} \tau_{g}^{\prime}\left(u_{0}\right)\left(u-u_{0}\right)^{2}+\cdots
$$

This ends the proof.

Proposition 5. Consider the boundaryless surface $\mathbf{S}_{\epsilon}$ obtained from a surface $\mathbf{S}$ by a parallel displacement of distance $\epsilon$ in both normal directions, glued to each other by the half tubes $\mathbf{T}_{\epsilon}$ of radius $\epsilon>0$ centered along the boundary $\mathbf{B}$ of $\mathbf{S}$. Then for small $\epsilon$, the surface $\mathbf{S}_{\epsilon}=\mathbf{S}_{\epsilon,+} \cup \mathbf{T}_{\epsilon} \cup \mathbf{S}_{\epsilon,-}$ is smooth outside the curves $\mathbf{B} \pm \epsilon N$ and regular of class $C^{1}$ along these curves.

The principal foliations lines of $\mathbf{S}_{\epsilon}$ are as follows:

1. The minimal principal lines of $\mathbf{S}_{\epsilon}$, away from tangencies, are the minimal principal lines of $\mathbf{S}_{\epsilon,+}$, together with the minimal principal lines of the tubes $\mathbf{T}_{\epsilon}$ (semi circles) and the minimal principal lines of the surface $\mathbf{S}_{\epsilon,-}$. These last mentioned curves are the parallel translation of the maximal principal ones of $\mathbf{S}$.

2. The maximal principal lines of $\mathbf{S}_{\epsilon}$, away from tangencies, are the maximal principal lines of $\mathbf{S}_{\epsilon,+}$, together with the maximal principal lines of the tubes $\mathbf{T}_{\epsilon}$ (curves defined by the differential equation $d \theta-\tau_{g} d u=0$ ) and the maximal principal lines of the surface $\mathbf{S}_{\epsilon,-}$. These last mentioned curves are the parallel translation of the minimal principal ones of $\mathbf{S}$. See Figure 3.

Proof. In this situation the tube $\mathbf{T}_{\epsilon}$ is glued to $\mathbf{S}_{\epsilon,+}$ at $\theta=0$ and to $\mathbf{S}_{\epsilon,-}$ at $\theta=\pi$. The tube is oriented positively with normal exterior and $\mathbf{S}_{\epsilon,-}$ has orientation opposite of $\mathbf{S}_{\epsilon,+}$. The minimal (respectively maximal) principal lines of $\mathbf{S}_{\epsilon,-}$ are obtained by parallel displacement of the maximal (respectively minimal) principal lines of $\mathbf{S}_{\epsilon,+}$.

The considerations above allow us to define the principal configuration of $\mathbf{S}_{\epsilon}$ as a pair of piecewise smooth foliations with singularities located at umbilics on $\mathbf{S}_{\epsilon} \backslash \mathbf{B}_{\epsilon}$ and the minimal 

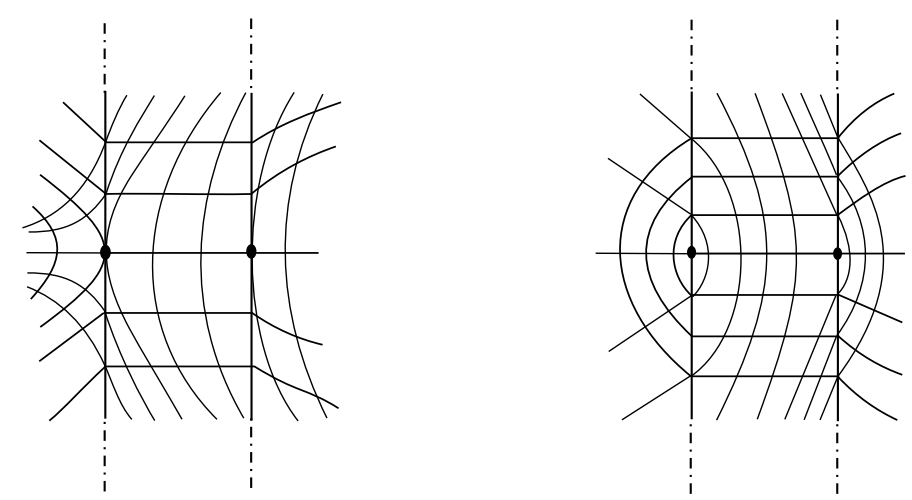

Fig. 3 - Piecewise Smooth Principal configuration of $\mathbf{S}_{\epsilon}$ : internal and external tangency.

(resp. maximal) tangencies with $\mathbf{B}_{\epsilon}$ when $\epsilon>0$ (resp. when $\epsilon<0$ ). The piecewise smooth leaves are defined by continuation of the principal lines on the tube and parallel surfaces, crossing through $\mathbf{S}_{\epsilon, \pm}$. In this context the external, $E$, and internal, $I$, minimal tangencies give rise to local configurations topologically equivalent to those around $D_{1}$ and $D_{3}$ umbilics.

Recall that the main result of this paper establishes that by a suitable smoothing the piecewise smooth principal configuration is deformed into a smooth one for which curves of "new" umbilics appear along arcs which are tangent to $E$ and $I$. Furthermore, the umbilics are Darbouxian of types $D_{1}$ and $D_{3}$, respectively. See Figures 2 and 3 for an illustration of the principal configuration in $\mathbf{S}_{\epsilon, \delta}$ and $\mathbf{S}_{\epsilon}$.

\section{SMOOTHING OF $S_{\epsilon}$ IN A LOCAL CHART}

In this section will be studied the principal configurations of the smoothing of the surface $\mathbf{S}_{\epsilon}$ by the operation of $\delta-$ smoothing. To this end consider an appropriate local chart $(u, v)$.

Proposition 6. Let $c:[0, l] \rightarrow \mathbb{R}^{3}$ be a parametrization by arclength $u$ of a connected component of $\mathbf{B}_{\epsilon}$ such that $\{T, N \wedge T, N\}$ is a positive frame of $\mathbb{R}^{3}$. Then the expression

$$
\beta(u, v)=c(u)+v(N \wedge T)(u)+\left(-\epsilon+\sqrt{\epsilon^{2}-v^{2}}\right) N(u)
$$

defines a regular parametrization of the tube $\mathbf{T}_{\epsilon}$ of radius $\epsilon$ centered at the curve $c-\epsilon N$.

Proof. Direct by the parametrization of the circle in the plane $\{N \wedge T, N\}$.

Proposition 7. Consider the surface $\mathbf{S}_{\epsilon}$ with boundary $\mathbf{B}_{\epsilon}$. Then the surface $\mathbf{S}_{\epsilon}$ has the following parametrization

$$
\begin{aligned}
& \beta_{\epsilon}(u, v)=c(u)+v(N \wedge T)(u)+R(u, v, \epsilon) N(u), \quad 0<v<\delta, \\
& \alpha_{\epsilon}(u, v)=c(u)+v(N \wedge T)(u)+S(u, v, \epsilon) N(u), \quad-\delta<v \leq 0,
\end{aligned}
$$


where,

$$
\begin{aligned}
& R(u, v, \epsilon)=-\epsilon+\sqrt{\epsilon^{2}-v^{2}}, \quad 0<v<\delta \\
& S(u, v, \epsilon)=k_{n}^{\perp} \frac{v^{2}}{2}+a(u, \epsilon) \frac{v^{3}}{6}+\cdots, \quad-\delta<v \leq 0 .
\end{aligned}
$$

Here $k_{n}^{\perp}=k_{n}^{\perp}(u, \epsilon)$ is the normal curvature of $\mathbf{B}_{\epsilon}=B+\epsilon N$ and $u$ is the arc length of $c$.

Proof. Similar to that of proposition 1.

REMARK 4. The principal curvatures of $\mathbf{S}$ and $\mathbf{S}_{\epsilon}$ are related by:

$$
k_{2}(\epsilon)=\frac{k_{2}}{1+\epsilon k_{2}}, \quad k_{1}(\epsilon)=\frac{k_{1}}{1+\epsilon k_{1}}
$$

The extensions of $\beta_{\epsilon}$ and $\alpha_{\epsilon}$, given by equation 6 , in a neighborhood of $c$, i.e., for $-\delta<v<\delta$, it will be supposed in the following.

Let $h$ be a smooth function defined in $\mathbb{R}$ satisfying the properties, $\left.h\right|_{-\infty, 0)}=1,\left.\quad h\right|_{\delta, \infty)}=0$ and $h$ decreasing in the interval $[0, \delta]$.

An example of such $h$ is given by the following.

Let

$$
\psi(v)= \begin{cases}0, & \text { for } \quad v \leq 0 \\ e^{-\frac{a}{v}}, & \text { for } \quad 0<v \leq \delta\end{cases}
$$

Define $h$ by,

$$
h(v)=\frac{\psi(\delta-v)}{\psi(v)+\psi(\delta-v)}
$$

Also consider the function

$$
H(v)=-1+h+2 v h_{v}+\frac{1}{2} v^{2} h_{v v}=-\left[(1-h) \frac{v^{2}}{2}\right]_{v v}
$$

that will appear in the proof of Theorem 1. See section 4 and equation 13.

Proposition 8. Consider the function $H(v)=-1+h+2 v h_{v}+\frac{1}{2} v^{2} h_{v v}$ defined in the interval $[0, \delta]$. Then for an appropriate transition function $h$ it follows that $H(v)<0$ for all $v \in(0, \delta]$.

Proof. Let

$$
\psi(v)= \begin{cases}0, & \text { for } \quad v \leq 0 \\ e^{-\frac{a}{v}}, & \text { for } \quad 0<v \leq \delta\end{cases}
$$

Define

$$
h(v)=\frac{\psi(\delta-v)}{\psi(v)+\psi(\delta-v)}
$$

Then $h$ is a $C^{\infty}$ function having all derivatives equal to zero at $v=0$ and $v=\delta$, with $h(0)=1, h(\delta)=0$ and $h$ being decreasing in the interval $[0, \delta]$. 
Plotting the graphs of $h$ and $H$ it follows that for $a>0$ small enough $h$ has the properties mentioned above and $\left.H\right|_{(0, \delta)}$ is negative. See Figure 4. This numerical assertion can be corroborated by an asymptotic analysis in $a>0$.

In fact, as $h$ is strictly decreasing in the interval $[0, \delta]$ and is concave near 0 , i.e., $h_{v v}(v)<0$, it follows that $H$ is strictly decreasing in an interval $[0, \rho(a, \delta)]$ with $H(0)=0$ and having all derivatives equal to zero at $v=0$.

The function $H$ has the following asymptotic expansion in $a$.

$$
H(v, a, \delta)=-\frac{1}{2}+\frac{1}{4} \frac{\delta^{3}-4 v \delta^{2}+4 v^{2} \delta-2 v^{3}}{v(\delta-v)^{3}} a+o\left(a^{2}\right)
$$

In a compact interval $[\rho(a, \delta), \delta]$ the function

$$
H_{a}(v)=\frac{1}{4} \frac{\delta^{3}-4 v \delta^{2}+4 v^{2} \delta-2 v^{3}}{v(\delta-v)^{3}}=\frac{1}{4}\left[\frac{1}{v}+\frac{v \delta-v^{2}-\delta^{2}}{(\delta-v)^{3}}\right]
$$

is negative if $\delta$ is small.

Therefore, it follows that for $a>0$ and $\delta>0$, both small, the function $H$ is negative in the interval $[0, \delta]$, as asserted.
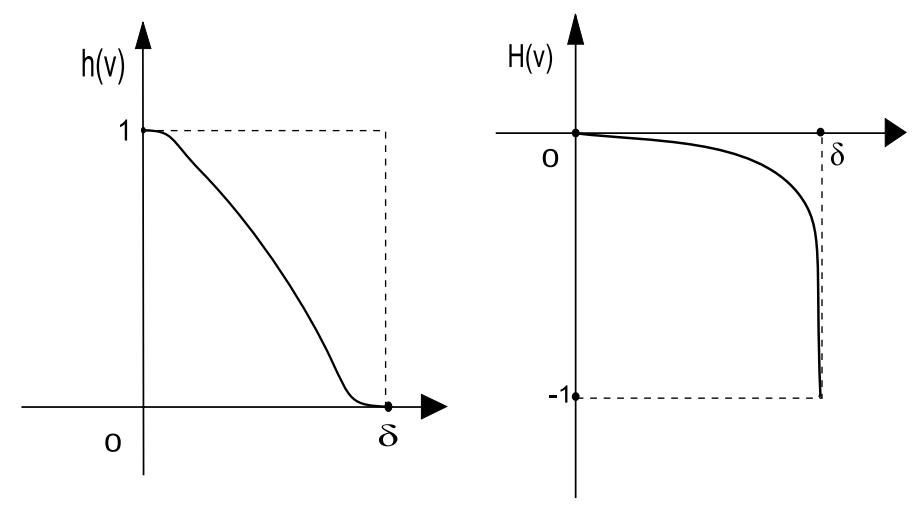

Fig. 4 - Transition function $h$ and function $H$.

REMARK 5. The function $H(v)=-\left[(1-h(v)) \frac{v^{2}}{2}\right]_{v v}$ is proportional to the curvature of the plane curve $C(v)=\left(v,(1-h(v)) \frac{v^{2}}{2}\right)$. For an appropriated $h$ as considered above $H$ is negative if $a>0$ is sufficiently small.

Consider the parametrization of the surface $\mathbf{S}_{\epsilon}$ given by equation 6 and consider the $\delta$-smoothing

$$
\gamma(u, v, \epsilon)=\alpha_{\epsilon}(u, v) h\left(\frac{v}{\epsilon^{2}}\right)+\left(1-h\left(\frac{v}{\epsilon^{2}}\right)\right) \beta_{\epsilon}(u, v), \quad-\delta<v<\delta .
$$


Direct calculation shows that

$$
\begin{aligned}
\gamma(u, v, \epsilon)= & c(u)+v(N \wedge T)(u) \\
& +\left\{h\left(\frac{v}{\epsilon^{2}}\right)\left[k_{n}^{\perp} \frac{v^{2}}{2}+a(u, \epsilon) \frac{v^{3}}{6}+\cdots\right]\right. \\
& \left.+\left[1-h\left(\frac{v}{\epsilon^{2}}\right)\right]\left[-\epsilon+\sqrt{\epsilon^{2}-v^{2}}\right]\right\} N(u)
\end{aligned}
$$

Now consider the rescaling $v=\epsilon^{2} \bar{v}$ in the expression above and rewriting back $(v=\bar{v})$, it is obtained that

$$
\begin{aligned}
\gamma(u, v, \epsilon)= & c(u)+\epsilon^{2} v(N \wedge T)(u)+A(u, v, \epsilon) N(u) \\
= & c(u)+\epsilon^{2} v(N \wedge T)(u) \\
& +\left\{\epsilon^{4} h(v)\left[k_{n}^{\perp} \frac{v^{2}}{2}+\epsilon a(u, \epsilon) \frac{v^{3}}{6}+\cdots\right]\right. \\
& \left.+\epsilon[1-h(v)]\left[-1+\sqrt{1-\epsilon^{2} v^{2}}\right]\right\} N(u) .
\end{aligned}
$$

The non unitary normal vector to $\mathbf{S}_{\epsilon}$ is given by

$$
\bar{N}=\left(N_{1}, N_{2}, N_{3}\right)=N_{1} T(u)+N_{2}(N \wedge T)(u)+N_{3} N(u)
$$

where, $N_{1}=\left(a_{2} b_{3}-a_{3} b_{2}\right) / \epsilon^{2}, N_{2}=\left(a_{3} b_{1}-a_{1} b_{3}\right) / \epsilon^{2}, N_{3}=\left(a_{1} b_{2}-a_{2} b_{1}\right) / \epsilon^{2}$ and

$$
\begin{aligned}
a_{1} & =1-\epsilon^{2} k_{g} v-k_{n} A & b_{1} & =0, \\
a_{2} & =-\tau_{g} A, & b_{2} & =\epsilon^{2}, \\
a_{3} & =A_{u}+\epsilon^{2} \tau_{g} v, & b_{3} & =A_{v} .
\end{aligned}
$$

Let

$$
\begin{array}{llrl}
a_{11}=\frac{\partial a_{1}}{\partial u}-k_{g} a_{2}-k_{n} a_{3}, & a_{21}=-\epsilon^{2} k_{g}-k_{n} A_{v}, & a_{31}=0, \\
a_{12}=\frac{\partial a_{2}}{\partial u}+k_{g} a_{1}-\tau_{g} a_{3}, & a_{22}=-\tau_{g} A_{v}, & a_{32}=0, \\
a_{13}=\frac{\partial a_{3}}{\partial u}+k_{n} a_{1}+\tau_{g} a_{2}, & a_{23}=\epsilon^{2} \tau_{g}+A_{u v}, & a_{33}=A_{v v} .
\end{array}
$$

Therefore,

$$
\begin{aligned}
& \gamma_{u u}=a_{11} T+a_{12} N \wedge T+a_{13} N \\
& \gamma_{u v}=a_{21} T+a_{22} N \wedge T+a_{23} N \\
& \gamma_{v v}=a_{31} T+a_{32} N \wedge T+a_{33} N
\end{aligned}
$$

The coefficients of the first fundamental form of $\gamma$ are given by:

$$
\begin{aligned}
& E=\left\langle\gamma_{u}, \gamma_{u}\right\rangle=a_{1}^{2}+a_{2}^{2}+a_{3}^{2} \\
& F=\left\langle\gamma_{u}, \gamma_{v}\right\rangle=a_{1} b_{1}+a_{2} b_{2}+a_{3} b_{3} \\
& G=\left\langle\gamma_{v}, \gamma_{v}\right\rangle=b_{1}^{2}+b_{2}^{2}+b_{3}^{2}
\end{aligned}
$$


The coefficients of the second fundamental form of $\gamma$ are proportional to the following functions:

$$
\begin{aligned}
& e=\left\langle\gamma_{u u}, \gamma_{u} \wedge \gamma_{v}\right\rangle=a_{11} N_{1}+a_{12} N_{2}+a_{13} N_{3} \\
& f=\left\langle\gamma_{u v}, \gamma_{u} \wedge \gamma_{v}\right\rangle=a_{21} N_{1}+a_{22} N_{2}+a_{23} N_{3} \\
& g=\left\langle\gamma_{v v}, \gamma_{u} \wedge \gamma_{v}\right\rangle=a_{31} N_{1}+a_{32} N_{2}+a_{33} N_{3}
\end{aligned}
$$

Direct calculation shows that:

$$
\begin{gathered}
E(u, v, \epsilon)=1-2 \epsilon^{2} k_{g} v+\epsilon^{3} k_{n}(1-h)+\epsilon^{4} v^{2}\left(\tau_{g}^{2}+k_{g}^{2}-k_{n} k_{n}^{\perp} h\right)+\epsilon^{6}(\cdots) \\
F(u, v, \epsilon)=\frac{1}{12} \epsilon^{5} v^{2}\left[\frac{1}{2} \tau_{g}\left(-1+h+v h_{v}\right)+\frac{1}{2} \epsilon \tau k_{n}^{\perp}\left(h+v h_{v}\right)+\epsilon^{2} v(\cdots)\right] \\
G(u, v, \epsilon)=\epsilon^{4}\left[1+\frac{1}{4} \epsilon^{2} v^{2}\left(v h_{v}+2 h-2\right)^{2}+\epsilon^{4} v^{2}(\cdots)\right] \\
e(u, v, \epsilon)=k_{n}+\epsilon k_{g} v\left(1-h-\frac{1}{2} v h_{v}\right) \\
\quad+\frac{1}{2} \epsilon^{2} v\left[2 \tau^{\prime}-k_{g}\left(4 k_{n}+2 k_{n}^{\perp} h+k_{n}^{\perp} v h_{v}\right)\right]+\epsilon^{3}(\cdots) \\
f(u, v, \epsilon)=\epsilon^{2} \tau_{g}+\epsilon^{4} v\left[\left(k_{n}^{\perp}\right)^{\prime} v\left(\frac{1}{2} v h_{v}+h\right)+\tau_{g} v^{2}\left(h-1+v h_{v}\right)^{2}\right]+\epsilon^{6}(\cdots) \\
g(u, v, \epsilon)=\epsilon^{3}\left[-1+h+2 v h_{v}+\frac{1}{2} v^{2} h_{v v}\right]+\epsilon^{4} k_{n}^{\perp}\left[h+2 v h_{v}+\frac{1}{2} v^{2} h_{v v}\right]+\epsilon^{5} v(\cdots)
\end{gathered}
$$

Therefore it is obtained:

$$
\begin{aligned}
L(u, 0, \epsilon) & =(F g-G f)(u, 0, \epsilon)=-\epsilon^{6} \tau_{g} \\
M(u, 0, \epsilon) & =(E g-G e)(u, 0, \epsilon)=\epsilon^{4}\left(k_{n}^{\perp}-k_{n}\right) \\
N(u, 0, \epsilon) & =(E f-F e)(u, 0, \epsilon)=\epsilon^{2} \tau_{g}
\end{aligned}
$$

It follows that:

$$
\begin{aligned}
L(u, v, \epsilon)= & \epsilon^{6}\left[-\tau_{g}+\epsilon^{2} v(\cdots)\right] \\
M(u, v, \epsilon)= & \epsilon^{3}\left[-1+h+2 v h_{v}+\frac{1}{2} v^{2} h_{v v}\right] \\
& +\epsilon^{4}\left[k_{n}^{\perp}-k_{n}+k_{n}^{\perp}\left(-1+h+2 v h_{v}+\frac{1}{2} v^{2} h_{v v}\right)\right]+\epsilon^{5} v(\cdots) \\
N(u, v, \epsilon)= & \epsilon^{2} \tau_{g}+\frac{\epsilon^{4}}{4} v\left[\left(4 h+2 v h_{v}\right)\left(k_{n}^{\perp}\right)^{\prime}+\tau_{g}\left(h_{v}^{2} v^{3}-4 v^{2} h_{v}+4 v^{2} h h_{v}\right.\right. \\
& \left.\left.-8 v h+4 v+4 v h^{2}-8 k_{g}\right)\right]+\epsilon^{5}(\cdots)
\end{aligned}
$$

\section{PROOF OF THE MAIN RESULT}

In this section the proof of Theorem 1 will be given. In what follows the classification of Darbouxian umbilic points will be reviewed. See (Darboux 1896) and (Gutierrez and Sotomayor 1982, 1991) for proofs. 
Let 0 be an umbilic point of a $C^{4}$ immersion $\alpha$ parametrized in a Monge chart $(x, y)$ by $\alpha(x, y)=(x, y, h(x, y))$, where

$$
h(x, y)=\frac{k}{2}\left(x^{2}+y^{2}\right)+\frac{a}{6} x^{3}+\frac{b}{2} x y^{2}+\frac{c}{6} y^{3}+O(4)
$$

The differential equation of principal curvature lines is given by:

$$
-\left[b y+P_{1}\right] d y^{2}+\left[(b-a) x+c y+P_{2}\right] d x d y+\left[b y+P_{3}\right] d x^{2}=0
$$

where $P_{i}, i=1,2,3$, represent functions of order $O\left(x^{2}+y^{2}\right)$.

Let

$$
\Delta_{P}=4 b(a-2 b)^{3}-c^{2}(a-2 b)^{2}
$$

Proposition 9 (Gutierrez and Sotomayor 1982, 1991). Under the conditions above suppose that the transversality condition $T=b(b-a) \neq 0$ holds and consider the following situations:

$$
\begin{aligned}
& \left.D_{1}\right) \quad \Delta_{P}>0 \\
& \left.D_{2}\right) \quad \Delta_{P}<0 \text { and } \frac{a}{b}>1 \\
& \text { D. }) \frac{a}{b}<1
\end{aligned}
$$

Then each principal foliation has in a neighborhood of 0 , one hyperbolic sector in the $D_{1}$ case, one parabolic and one hyperbolic sector in $D_{2}$ case and three hyperbolic sectors in the case $D_{3}$. The umbilics are called Darbouxian of types $D_{1}, D_{2}$ and $D_{3}$.

See Fig. 5 for illustrations of $D_{1}$ and $D_{3}$. The type $D_{2}$ does not appear in this work.
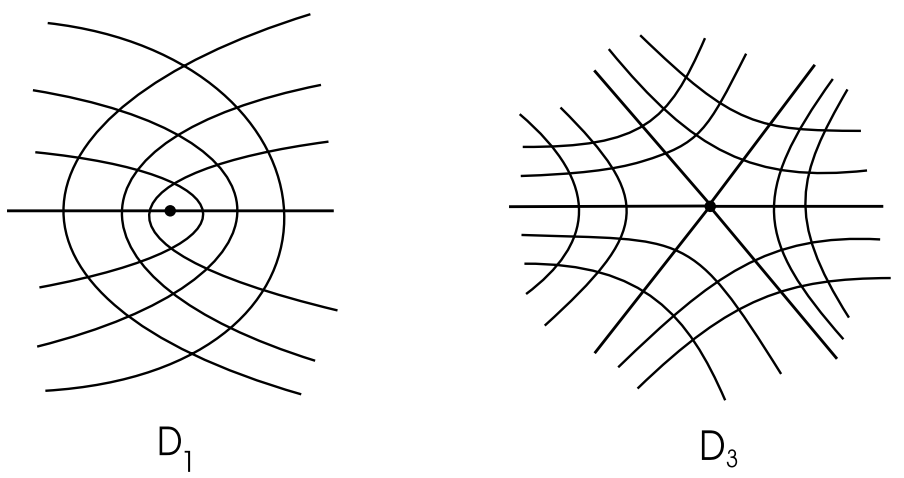

Fig. 5 - Darbouxian umbilic points $D_{1}$ and $D_{3}$.

LEMMA 1. Let $u_{0}$ be a point of tangency such that $\tau_{g}\left(u_{0}\right)=0, \tau_{g}^{\prime}\left(u_{0}\right) \neq 0$ and $\left(k_{n}^{\perp}-k_{n}\right)\left(u_{0}\right)>0$. Then the set $\mathcal{U}_{\epsilon}=\{(u, v, \epsilon): M(u, v, \epsilon)=N(u, v, \epsilon)=0\}$ is a regular curve. The curve of umbilic points $\gamma\left(\mathcal{U}_{\epsilon}\right)$ is tangent to the normal vector $N\left(c\left(u_{0}\right)\right)$ and is transversal to the surface $\mathbf{S}_{\epsilon, \delta}$ in a neighborhood of this point of tangency. 
Proof. Solving the equation $N(u, v, \epsilon)=0$ it follows, by Implicit Function Theorem, that there exists a smooth function $u(v, \epsilon)$ such that $N(u(v, \epsilon), v, \epsilon)=0$ and

$$
u(v, \epsilon)=\epsilon^{2} \xi(v, \epsilon), \quad \xi(0)=0 .
$$

Therefore the equation $M(u, v, \epsilon)=0$ is such that $M(u(v, \epsilon), v, \epsilon)=M(v, \epsilon)=0$. Applying the Implicit Function Theorem to this equation it follows that there exists a smooth function of the form $\epsilon=\varphi(v)$, where

$$
\varphi(v)=\frac{\left(1-h-2 v h_{v}-1 / 2 v^{2} h_{v v}\right)}{\left(k_{n}-k_{n}^{\perp}\right)\left(u_{0}\right)}[1+\cdots]
$$

with $M(v, \varphi(v))=0, \varphi(0)=0$ and is a flat function at $v=0$. By the properties of the transition function $h$, it follows that $\varphi$ is decreasing for $v>0$ small. So it follows that $v=\varphi^{-1}(\epsilon)$.

Returning to the original coordinates $(u, v, \epsilon)$, recalling that $v=\epsilon^{2} \bar{v}$ and so $\bar{v}=\varphi^{-1}(\epsilon)$, it follows that $v=\epsilon^{2} \varphi^{-1}(\epsilon)$. Therefore the curve of umbilic points is given by

$$
\mathcal{U}(\epsilon)=\left(\epsilon^{2} \xi\left(\epsilon^{2} \varphi^{-1}(\epsilon), \epsilon\right), \epsilon^{2} \varphi^{-1}(\epsilon), \epsilon\right) .
$$

Direct calculation gives $\mathfrak{U}^{\prime}(0)=(0,0,1)=N\left(c\left(u_{0}\right)\right)$.

Proposition 10. Suppose that $\tau_{g}(0)=0, \tau_{g}^{\prime}(0) \neq 0$ and $\left(k_{n}^{\perp}-k_{n}\right)(0)>0$. Then the curve of umbilic points $\gamma\left(\mathcal{U}_{\epsilon}\right)$ is tangent to the normal vector $N\left(c\left(u_{0}\right)\right)$ and is transversal to the surface $\mathbf{S}_{\epsilon, \delta}$ in a neighborhood of this point of tangency $c\left(u_{0}\right)$. This intersection point is an umbilic point of type $D_{1}$ or $D_{3}$ according to $\tau_{g}^{\prime}(0)<0$ (external tangency) or $\tau_{g}^{\prime}(0)>0$ (internal tangency). The principal configuration is regular near $c(0)-\epsilon N$.

Proof. The umbilic points of the surface $\gamma$ are given by equation $13, L(u, v, \epsilon)=M(u, v, \epsilon)=$ $N(u, v, \epsilon)=0$.

By Lemma 1 , near an umbilic point $\left(0, v_{1}\right)$, it follows that

$$
\begin{aligned}
L(u, v, \epsilon) & =-\epsilon^{6} \tau_{g}^{\prime}(0) u+o(2) \\
M(u, v, \epsilon) & =\epsilon\left(k_{n}^{\perp}-k_{n}\right)^{\prime}(0) u+b(\epsilon)\left(v-v_{1}\right)+o(2), \quad b(0)=b_{0}<0 \\
N(u, v, \epsilon) & =\tau_{g}^{\prime}(0) u+o(2)
\end{aligned}
$$

where $b_{0}=\frac{\partial H}{\partial v}\left(v_{1}\right)<0$.

The differential equation of curvature lines is given by,

$$
\left[-\tau_{g}^{\prime}(0) \epsilon^{2} u+o(2)\right] d v^{2}+\epsilon^{2}\left[\epsilon k_{0} u+b(\epsilon)\left(v-v_{1}\right)+o(2)\right] d u d v+\left[\tau_{g}^{\prime}(0) u+o(2)\right] d u^{2}=0
$$

where $k_{0}=\left(k_{n}^{\perp}-k_{n}\right)^{\prime}(0)$.

By the classification of Darbouxian umbilic points, Proposition 9, it follows that the tangent to the umbilic separatrices are defined by $u=\lambda v$, where

$$
\lambda\left[\tau_{g}^{\prime}(0) \lambda^{2}+\epsilon^{3} k_{0} \lambda+\epsilon^{2}\left(b-\tau_{g}^{\prime}(0) \epsilon^{2}\right)\right]=0 .
$$


Therefore the discriminant of the equation above is given by

$$
\Delta=\epsilon^{2}\left[-4 b \tau_{g}^{\prime}(0)+4 \epsilon^{2}\left(\tau_{g}^{\prime}(0)\right)^{2}+\epsilon^{4} k_{0}^{2}\right]
$$

So, if $\tau_{g}^{\prime}(0)>0$ it follows that $\Delta>0$ and the umbilic point is of the type $D_{3}$.

On the other hand, if $\tau_{g}^{\prime}(0)<0$ it follows that $\Delta<0$ and the umbilic point is of the type $D_{1}$. Near the point $c(0)-\epsilon N$, since the orientation is exchanged it follows that $k_{n}^{\perp}(0, \epsilon)-k_{n}(0, \epsilon)<0$ and then $M(u, v, \epsilon) \neq 0$ in a neighborhood of this point. Therefore the principal foliations are regular there.

REMARK 6. The main result of this paper shows that from a quadratic tangency the Darbouxian umbilic of type $D_{2}$ does not appear in the process of thickening and smoothing.

\section{CONCLUDING REMARKS}

A global result, relating quadratic tangencies, Darbouxian umbilic points and the Euler-Poincaré characteristic of the surface is given by the following proposition.

Proposition 11. Consider a compact, oriented surface $\mathbf{S}$ with regular boundary $\mathbf{B}$ such that all umbilic points of $\mathbf{S}$ are Darbouxian and the tangencies, internal and external, of the principal foliations $\mathcal{F}_{1 \mathbf{S}}$ and $\mathcal{F}_{2 \mathbf{S}}$ with the boundary at $\mathcal{C}_{\mathbf{S}}$ are quadratic. Then the following expression for the Euler-Poincaré characteristic for $\mathbf{S}$ and $\mathbf{S}_{\epsilon, \delta}$ holds:

$$
\chi\left(\mathbf{S}_{\epsilon, \delta}\right)=2 \chi(\mathbf{S})=2\left[\#\left(D_{1}\right)+\#\left(D_{2}\right)-\#\left(D_{3}\right)+\frac{\#(E)-\#(I)}{2}\right],
$$

where $\#\left(D_{i}\right), i=1,2,3$, is the number of umbilic points of type $D_{i}$ and $\#(E), \#(I)$ are, respectively, the number of external and internal tangencies of both principal foliations with the boundary $\mathbf{B}$.

Proof. The proof follows recalling that $\chi\left(S_{\epsilon, \delta}\right)=\chi\left(S_{\epsilon}\right)=2 \chi(S)$ and that $\chi\left(\mathbf{S}_{\epsilon, \delta}\right)$, by PoincaréHopf Theorem, is equal to the sum of the indices of the singularities of principal curvature line field $\mathcal{L}_{1 \mathbf{S}}$, see (Spivak 1980) and also (Garcia et al. 2000), and that near a point of quadratic tangency bifurcate a Darbouxian umbilic point of type $D_{1}$ or $D_{3}$, according the tangency is external or internal.

\section{ACKNOWLEDGMENTS}

The first author was partially supported by FAPESP, Grant 00/13571-6 and FUNAPE/UFG. Both authors are fellowship of CNPq. This work was done under the project PRONEX/FINEP/MCT Conv. 76.97.1080.00 - Teoria Qualitativa das Equações Diferenciais Ordinárias. 


\section{RESUMO}

Neste trabalho são estudadas as linhas de curvaturas principais de uma superfície com bordo e da superfície regular, sem bordo, obtida pelo processo de engrossamento e regularização. É analisada a relação entre as tangências quadráticas das folheações principais com o bordo e os pontos umbílicos Darbouxianos, da superfície sem bordo, que bifurcam dos referidos pontos de tangências.

Palavras-chave: configuração principal, pontos umbílicos, singularidade tangencial.

\section{REFERENCES}

Darboux G. 1896. Leçons sur la Théorie des Surfaces, vol. IV. Sur la forme des lignes de courbure dans la voisinage d'un ombilic, Note 07, Paris: Gauthier Villars, 420p.

Garcia R AND Sotomayor J. 1997. Structural stability of parabolic points and periodic asymptotic lines, Matemática Contemporânea, 12: 83-102.

Garcia R and Sotomayor J. 2000. Lines of Axial Curvature on Surfaces Immersed in $\mathbb{R}^{4}$, Differential Geometry and its Applications, 12: 253-269.

Garcia R AND Sotomayor J. 2001. Periodic behavior of principal curvature lines on surfaces with boundary, in preparation.

Garcia R, Gutierrez C and Sotomayor J. 2000. Lines of Principal Curvature around Umbilics and Whitney Umbrellas, Tohoku Math J, 52: 163-172.

Gutierrez C and Sotomayor J. 1982. Structural Stable Configurations of Lines of Principal Curvature, Asterisque, 98-99: 185-215.

Gutierrez C and Sotomayor J. 1991. Lines of Curvature and Umbilic Points on Surfaces, Brazilian $18^{\text {th }}$ Math. Coll., Rio de Janeiro: IMPA, 112p. Reprinted as Structurally Configurations of Lines of Curvature and Umbilic Points on Surfaces, Lima: Monografias del IMCA.

Gutierrez C And Sotomayor J. 1998. Lines of Curvature, Umbilical Points and Carathéodory Conjecture, Resenhas IME-USP, 3: 291-322.

Melo W and Palis J. 1982. Geometric Theory of Dynamical Systems, New York: Springer Verlag, 320p.

Sotomayor J And Teixeira M. 1998. Regularization of Discontinuous Vector Fields, International Conference on Differential Equations (Equadiff 95), Word Scientific Publishing Co. p. 207-223.

SpIVAK M. 1980. A Comprehensive Introduction to Differential Geometry, Vol. III, Berkeley: Publish or Perish, 530p. 